\title{
KONGRESSKALENDER
}

\section{März 2021}

\section{ABGESAGT}

03.-05.03.2021, Lippstadt-Eickelborn 36. Eickelborner Fachtagung zu Fragen der Forensischen Psychiatrie

Dr. Wallenstein

Kontakt: Eickelborner.Fachtagung@lwl.org

Information: www.forensik-lippstadt.de

\section{April 2021}

\subsubsection{1, Stadtroda}

Forensische Tagung: Persönlichkeitsstörungen und Delinquenz.

Asklepios Fachklinikum

Dr. R. Sternitzke

Information: r.sternitzke@asklepios.com

\section{Mai 2021}

14.05.2021, Dresden

25. Dresdner Frühjahrstagung

für Forensische Psychiatrie

Theorie und Praxis der forensischen

Psychopathologie - Zum 100. Geburtstag von Werner Janzarik

Dr. Lange, Prof. Dr. Bauer, Dr. Lammel, Dr. Sutarski

Information: www.forensik-dresden.de

19.-21.05.2021, Zürich, Schweiz

12. Internationales Symposium

Forensische Psychiatrie

World Trade Center Zürich

PD Dr. A. Rossegger, Prof. Dr. J. Endrass,

A. Nägeli, Prof. Dr. M. Graf

Information: www.forensiktagung.ch

\section{Juni 2021}

22-25.06.2021, Krakau, Polen 18th Annual IAFMHS Conference Past, Present, and Future of Forensic Mental Health Services

Information: www.iafmhs.org

25.06.2021, Berlin-Mitte

24. Berliner Junitagung für Forensische Psychiatrie und Psychologie Die erheblich verminderte Schuldfähigkeit \$ 21 StGB - Wie lässt sie sich erfahrungswissenschaftlich begründen?

Forensische Psychiatrie Psychologie Berlin e. V. (FPPB) \& Institut für Forensische Psychiatrie der Charité

Langenbeck-Virchow-Haus

Information: www.forensik-berlin.de

(Veranstaltungen)

\section{August 2021}

24-27.08.2021, Frankfurt am Main 16th Conference International Association for the Treatment of Sexual Offenders (IATSO)

Prof. Dr. Martin Rettenberger

Information: www.iatso.org

\section{September 2021}

02.-04.09.2021, Hannover

17. Fachtagung der Kriminologischen Gesellschaft (KG)

Kriminalität und Digitalisierung Digitalisierung und Kriminalität Tagungszentrum Schloss Herrenhausen Prof. Dr. Bliesener www.kfn.de/KrimG21
08.-11.09.2021, Bukarest, Rumänien European Association of Criminology 19th Annual ESC Conference

(Il)legal organizations and crime Information: http://www.esc-eurocrim.org/

29.09-02.10.2021, USA

Association for the Treatment of Sexual Abusers (ATSA)

40th Annual Research and Treatment Conference

Information: http://www.atsa.com/ future-atsa-conferences

\section{Oktober 2021}

08.10.2021, München

36. Herbsttagung für Forensische Psychiatrie

Abteilung für Forensische Psychiatrie der Universität München

Prof. Dr. K. Schiltz

Information: www.forensik-muenchen.de

Hinweise für den Kongresskalender bitte an: mail@hlkroeber.de

Auch bei diesem Kongresskalender stehen die Termine im besonderen Maße unter dem Vorbehalt von Verlegungen und Absagen 